\title{
An Ontology-Driven Framework and Web Portal for Spatial Decision Support
}

\author{
Naicong $\mathrm{Li}^{1}$, Robert Raskin ${ }^{2}$, Michael Goodchild ${ }^{3}$, Krzysztof Janowicz $^{3}$ \\ ${ }^{1}$ The Redlands Institute \\ University of Redlands \\ Redlands, CA USA 92373 \\ naicong_li@spatial.redlands.edu \\ ${ }^{2}$ Jet Propulsion Laboratory \\ Pasadena, CA USA 91109 \\ raskin@jpl.nasa.gov \\ ${ }^{3}$ Department of Geography \\ University of California \\ Santa Barbara, USA, CA USA 93106-4060 \\ good@geog.ucsb.edu \\ jano@geog.ucsb.edu
}

\section{Short title: Ontology-Driven Portal for Spatial Decision Support}

Keywords: spatial decision support, ontology, semantic portal, semantic search

The authors dedicate this article to the memory of co-author Rob Raskin, who passed away prior to its publication. Rob's research at the NASA Jet Propulsion Laboratory encompassed the fields of atmospheric science and GIScience. He was also interested in semantic interoperability and the development of common ontologies to foster sharing heterogeneous scientific data across knowledge communities. He founded the well-known Semantic Web for Earth and Environmental Terminology (SWEET), a modular and extensible set of upper-level ontologies for Earth system science. Rob was a founding member of the Spatial Decision Support (SDS) Consortium and a major contributor to the development to the SDS ontology. His presence will be deeply missed by colleagues in many fields to which he has made significant contributions.

Corresponding author: Naicong Li, Redlands Institute, University of Redlands, 1200 East Colton Avenue, PO Box 3080, Redlands, CA 92373, naicong_li@spatial.redlands.edu 


\title{
An Ontology-Driven Framework and Web Portal for Spatial Decision Support
}

\begin{abstract}
Numerous systems and tools have been developed for spatial decision support (SDS), but they generally suffer from a lack of re-usability, inconsistent terminology, and weak conceptualization. We introduce a collaborative effort by the SDS Consortium to build a SDS knowledge portal. We present the formal representation of knowledge about SDS, the various ontologies captured and made accessible by the portal, and the processes used to create them. We describe the portal in action, and the ways in which users can search, browse, and make use of its content. Finally, we discuss the lessons learned from this effort, and future development directions. Our work demonstrates how ontologies and semantic technologies can support the documentation and retrieval of dynamic knowledge in GIScience by offering flexible schemata instead of fixed data structures.
\end{abstract}




\section{Introduction and Motivation}

Spatial decision support (SDS) provides computational or informational assistance for making better-informed decisions about problems with a geographic or spatial component. This support assists with the development, evaluation, and selection of proper policies, plans, scenarios, projects, interventions, or solution strategies (SDS Consortium 2008). SDS plays an ever-increasing role in planning and decision making when solving complex, large-scale problems in GIScience. Numerous spatial decision support systems (SDSS) and tools have been developed to date. With few exceptions, these systems and tools have been built independently for each application, are not easily reusable, are not readily adaptable to changing business environments, and their performance is not independently verifiable. The lack of modularity represents a major blockage for their re-usability and interoperability (Goodchild and Glennon 2008).

Developing reusable and interoperable SDS systems and tools depends on a high level of understanding of the domain of spatial decision support, including the identification of the fundamental concepts of SDS. Such an understanding is difficult to achieve for several reasons. The knowledge in the field of SDS is vast, spanning many areas including: spatial decision processes and their steps (Malczewski 1999), methods and techniques used during a spatial decision process (Malczewski 1999, 2006); participation and collaboration dimensions of the decision process (Armstrong 1993, Jankowski and Nyerges 2001, Sieber 2006, Jankowski et al. 2006); systems functionality (Malczewski 1999, Densham 1991); and the data, data models, and process models needed to solve a decision problem in an application domain. In addition to the challenge of integrating information from many aspects of SDS, multiple overlapping fields of study have been developed by various research communities. Examples include SDSS, planning support systems (PSS) (Batty 2008), group decision making (Jankowski and Nyerges 2001), public participation geographic information systems (Sieber 2006), intelligent spatial decision support systems (Leung 1997), and spatial expert support systems (Zhu and Healey 1992). Methods and tools developed in these fields of study often overlap or partially overlap but may use the same terms to refer to different concepts or use different terms to refer to the same concept. Therefore, experts may assume that their models are based on the same assumptions and will interoperate when they are not commensurable and combining them may lead to misleading results (Harvey 1999). This 
semantic mismatch inhibits the mutual understanding and sharing of information and experiences about SDS development. Additionally, a common conceptual framework for synthesizing and presenting this vast body of knowledge is missing. These deficiencies make it difficult and error-prone to select appropriate methods and tools and other SDS resources, understand their implications, and combine them into a solution strategy.

In this paper, we present the results of a large-scale project that aims to capture the body of knowledge in SDS using a semantic-enabled and ontology-driven Web portal. The presented work serves as a source of information and a common vocabulary for SDS researchers and practitioners. It provides learning material for students and newcomers interested in this multidisciplinary field. It creates a standard way to specify and register SDS resources and thus helps to avoid semantic mismatches among them. In doing so, the presented system also contributes to the vision of a semantics-enabled e-Science (De Roure and Hendler 2004). The SDS ontologies are the result of collaborative work of the SDS Consortium (SDS Consortium 2011), whose 33 members include scholars and practitioners in SDS and related fields from various universities, NGOs, and government agencies, with application domains spanning natural and human systems. Our work highlights how semantic technologies and ontologies can be used to model knowledge in a complex and heterogeneous domain, improve documentation and retrieval, and ease the development of flexible Web applications. The presented portal is automatically generated from the SDS ontologies; consequently, all aspects of SDS-related information ranging from process workflows, methods and tools to case studies can be managed using a common framework. New data at the schema and instance levels can be integrated on-the-fly without hard-coded changes to the portal. The ability to move the business logic from the application code to the data is one of the strengths of the semantic approach and a prerequisite to handle dynamic information. The lessons learned in creating such portal and ontologies go beyond SDS but can be adapted to improve the understanding of and access to geographic information analysis, in general.

The remainder of this paper is organized as follows. Section 2 presents the content analysis of the body of knowledge in SDS, and introduces the SDS ontology that we have developed to capture this knowledge. In Section 3, we present the SDS Knowledge Portal, a semantic-enabled Web application that provides the user with easy access to SDS-related knowledge and resources. Section 4 illustrates the usage of the portal through two use cases. Section 5 discusses lessons learned. Finally, Section 6 concludes our work and points to future research directions. 


\section{Formal Representation of the Body of Knowledge in SDS}

Developing a fine-grained, highly formalized ontology for a vast body of knowledge and resources is challenging, especially with limited resources. However, we can create a sound conceptual framework for organizing this knowledge, fill it initially with essential content, and let the content develop further over time. This conceptual framework should ease information retrieval and navigation and be extendable in the future. In this work, we present a set of domain-specific ontologies, which serve as the conceptual framework to organize SDS-related knowledge and resources. These ontologies have been agreed upon by the SDS Consortium members and have been developed to satisfy five major objectives: 1) document knowledge in a formal and consistent way that limits the interpretation of the used terms towards their intended meaning; 2) establish a standard against which SDS resources can be classified and registered; 3 ) ease retrieval and browsing through this vast body of knowledge; 4) uncover inconsistencies and incompatibilities; and 5) reduce the barriers of entry to the successful application of SDS.

\subsection{The Content of the SDS Ontologies}

The SDS ontologies currently include definitions for over 900 concepts, 500 instances, 200 attributes and relations, all provided with a common model for labels, synonyms and acronyms, detailed descriptions, and provenance information. ${ }^{1}$ The ontologies are coded in OWL (Web Ontology Language). For the purpose of conceptual clarity as well as following the best practice in ontology development, the concepts are partitioned logically into over 40 ontologies. These ontologies are then grouped into several major components for the ease of concept browsing and search (see Section 3): decision problem types, planning/decision process phases and steps, methods and techniques, technology, domain data and knowledge, people and participation, and resources for decision processes. A rich set of relations connects the concepts in and across these components, which could be used to guides the decision maker to best practices in assembling a decision solution strategy.

The decision problem type ontology includes subcategories such as impact assessment, suitability assessment, location allocation, site search or selection, network design, and scheduling. Besides taxonomic relations, several attributes and relations describe decision problem types, such as spatial scale, temporal extent, and various decision contexts defined in

The terms class and concept as well as relation and role are used synonymously in the Semantic Web community. 
the decision context ontology, including institutional, legal, social, cultural, geographic contexts and application domains.

The planning and decision process ontology describes structured or semi-structured decision process workflows with their phases and steps. Classical decision theories (Simon 1960) divide the decision process into a few major phases, such as intelligence, design, and choice. There are many variations on this sequence (Malczewski 1999, Steinitz 1990), for example, phases in some of the approaches are considered sub-steps in other variations. Example decision process phases include problem identification, stakeholder engagement, process mapping, condition assessment of the current state of the system, design of alternative solutions, evaluation of the alternative designs including impact analysis, and selection of a design alternative based on a set of pre-defined criteria. Associated with these phases and steps are commonly used methods and techniques, decision participant types and roles, and expected outcomes. Depending on the nature of the decision problem type and its application domain, the decision process may adopt a workflow that is most suitable for its purpose.

A separate ontology contains a large set of SDS methods and techniques organized into types and subtypes based on their purpose, such as spatial modeling, simulation, optimization, multi-criteria decision analysis, and consensus building. They are defined with a set of parameters such as inputs and outputs, and linked with specific decision process steps where they are commonly used.

The SDS technology ontology defines those concepts necessary to classify SDS software in terms of their system functionality, such as data management, tools/models management, scenario management, process flow control, collaboration support, report generation, and visualization.

The domain data and knowledge ontology includes definitions of relevant data attributes, data source attributes, data topics, knowledge domains to describe and classify data sources, data models, and process models.

The people and participation ontology defines concepts related to participatory and collaborative planning and decision making as well as the various participant roles during various steps of a decision process.

The resources ontology defines SDS resource types such as references to resource instances including workflow templates, methods and techniques, tools, models, case studies, data sources, literature, and related Web sites. These resources are defined and classified with 
extensive use of the concepts and attributes defined in other branches of the conceptual framework described above.

As of Spring 2012, the knowledge base spans more than 9 decision process workflows with their phases and steps, about 100 methods, 80 SDS tools and models, various data sources and case studies, and more than 700 publications related to SDS research.

\subsection{The SDS Ontology Development Process}

The process of developing SDS ontologies is iterative and ongoing. Our first task was to identify the relevant concepts to be included in the ontology. For each concept, we identified the set of attributes and relations with other concepts needed to make the structure of the body of knowledge explicit and to facilitate navigation and retrieval. For example, implements (and its inverse relation implemented by) is needed to present the relations between SDS methods and software tools which realize these methods.

Two specialist workshops were held in 2008, engaging well-known scholars, experienced practitioners, technology developers, and ontology experts. The outcomes of these workshops included: 1) Major ontologies were identified, to partition SDS concepts into branches of knowledge that act as the top nodes of the Web portal; 2) The modular design of the SDS ontologies was finalized, and the inter-dependencies of ontologies were identified; 3) The collaborative discussion and debates during the workshops helped deepen a common understanding of the structure of the body of knowledge, including determining the set of attributes and relations needed for some important facets, such as decision making phases; 4) The diverse backgrounds of the participants brought together multiple perspectives on SDS that were methodologically varied and domain specific. These contributions are essential for the development of ontologies whose aim is to include and synthesize all aspects of SDS. At the end of each workshop, participants formed focus groups, each refining a component of the SDS ontologies.

The ontology development process was mostly top-down, in that its design was driven by an overall understanding of the structure of SDS research. Some of the key concepts considered essential ingredients for such understanding (e.g., the decision process, methods, and their inter-relations) drove the development of other supporting concepts. However, a bottom-up, data-driven approach was adopted in a few cases. For instance, we imported a set of SDS tools from an existing database and came across some describing attributes that we had not considered before, but subsequently included. 
While the consortium's development model resembles METHONTOLOGY (Fernandez 1997) and related approaches, our maintenance phase differs. The workshops set the ground for major changes and releases. In contrast, small changes requested by members are all handled, formalized, and implemented by a single main editor to ensure consistency in the used engineering paradigms and ontological commitments. In the future, we may introduce a collaborative Web-platform to assist members in contributing OWL code directly; however, the size of the consortium may require a rigid framework for such contributions.

\subsection{Design Considerations of the SDS Ontologies}

The design of the SDS ontologies is purpose-driven. Besides capturing the structure of knowledge in SDS, ontology development helps facilitate access to information. For instance, the ontologies support users by providing definitions of essential concepts related to SDS as well as access to SDS resources. This purpose has dictated several design choices.

One of the design considerations is scope. SDS is a research and application area that cuts across academic disciplines and human knowledge domains. While a basic understanding of these domains is necessary to classify the functions of the SDS tools and models, developing ontologies for these domains is beyond the scope of our project. We have focused on defining the essential concepts in SDS, including those for decision problem types, decision context, decision process, methods, technology, participation and collaboration, and decision support resources. These concepts reside in a set of SDS core ontologies but we also refer to external concepts, for example, to link to data attributes that specify the inputs and outputs of tools and models. These external concepts reside in a set of supporting ontologies. We have initially included only those supporting concepts that are directly referred to by core concepts and their definitions, and in contrast to the SDS core, their definitions merely consist of natural-language descriptions.

Another design consideration is the degree of formalization: that is, which attributes and relations are minimal but sufficient for the automation of information access and the required reasoning support. For instance, when we define the steps in a decision process, significant information about those steps is provided through natural-language definitions. Formalized properties include sub-steps, methods and techniques commonly used for these steps, tools that support them, participant types, among others. Which information should be described in natural language and which should be formalized depends on the relations to be established 
between concepts. This choice, in turn, is determined by the navigation needs of the SDS portal; see Section 3.

Inverse properties are heavily used in the SDS ontologies. As mentioned above, SDS methods have an implemented-by relation with tools and models, and tools and models have an implements relation with methods. Inverse relations significantly improve navigation and ease the development of the portal (most inverse relations are automatically inferred).

In some cases we had to make a choice between modeling an entity as a class or instance. For example, specific methods are currently represented as classes instead of instances. This approach ensures that our ontologies are extendable and can grow with the research field. Refinements to existing SDS methods can be modeled as subclasses. For example, for sensitivity analysis method is an area of active research, and variations have been developed in recent years (Ligmann-Zielinska and Jankowski 2008). These submethods are represented as subclasses of sensitivity analysis. This flexibility on the schema level is an important feature as new knowledge can be integrated easily. Subsuming reasoning-based query expansion ensures that users can navigate the portal from generic to more specific SDS methods; see Section 3.

Another common design decision was to choose between taxonomic and non-taxonomic relations. To keep the navigation interface clear and to comply with the literature, we identified a minimal set of subclasses and superclasses, and expressed as many other facts as possible using non-taxonomic relations or attributes. For example, the SDS tools are instances of various subclasses of the software class. They also have many properties describing their relation to other branches of the SDS ontologies, such as methods and decision process steps. Although tools are formally coded as having one taxonomic relation to the class software type, users may want to browse tools differently. For example, one may want to have the tools organized based on what decision process activity types they support. This structure requires a tool taxonomy based on these decision process activity types. Therefore, before each ontology version release, we automatically derive an additional subsumption relationship for the tools keyed off a decision process activity type, derived from the each tool's relation to decision process steps. Consequently, on the Web portal, the tools can be browsed either through a decision process activity type tools taxonomy or a software type taxonomy.

As with the consideration for any systems design, we have been mindful to maintain modularity in the ontology design, and partitioned the related concepts into a set of SDS 
ontologies. The dependencies among these ontologies were carefully considered, so that supporting ontologies containing concepts that are more generic (upper level) are imported into ontologies that are more SDS-domain specific, but not the other way around. Besides being conceptually cleaner, this practice allows easy import of well-established third-party ontologies and makes our ontologies reusable for external parties. For instance, the dataattributes ontology imports a data-topic ontology based on the ISO 19115 data-topic categories. Several of the supporting ontologies developed by the SDS Consortium ultimately should be replaced by domain- or application-level ontologies developed by other expert groups. For example, we plan to replace part of the data-attributes ontology by importing the relevant Semantic Web for Earth and Environmental Terminology (SWEET) ontologies (Raskin and Pan 2005). SWEET provides support for scientific and numerical concepts, such as scientific units, scientific relations, provenance, and data representation. We believe that ontologies should carry as few ontological commitments as possible and interlink with other Semantic Web ontologies by matching and alignment (Shvaiko and Euzenat 2008) whenever knowledge has to be added that exceeds our own expertise.

\section{SDS Ontologies in Action: the SDS Knowledge Portal}

SDS ontologies could potentially benefit a diverse set of user communities, including SDS practitioners, researchers and students, SDS resource providers, decision makers in various application domains, and members of general public who are interested in this subject. To make the content of the SDS ontologies easily accessible initially by the research and practice community, a portal was launched in 2009 and extended in 2011. The content of the SDS Knowledge Portal is entirely driven by the SDS ontologies as proposed by the SEAL (SEmantic portal) approach (Maedche et al. 2003). The portal serves a dual purpose: accessing the body of knowledge in SDS, and accessing the resources which are registered and characterized by the ontologies, e.g., tools, models, data sources, and case studies. Figure 1 displays a typical page from the portal with callout boxes indicating the main components and functionalities for ontology and resource browsing as well as the semantic search (see more detailed descriptions in sections 3.2 and 3.3 below).

(Figure 1 about here) 
Faceted search as an exploratory, multi-filter paradigm has been proposed for browsing semantic-enabled portals (Suominen et al. 2007). While this approach is feasible, we instead combined several query paradigms, some of which resemble facets. The SDS portal was developed with a heterogeneous user base in mind. Browsing by category or directly navigating the ontology graph may be preferred by domain experts and learners of SDS, while users in search of appropriate methods and tools for their work may prefer direct access via semantic search to arrive at a specific documentation.

In the following, we present the system architecture of the SDS Knowledge Portal, the main functionality, and how the portal is generated from the ontologies introduced above.

\subsection{System Architecture of the SDS Knowledge Portal}

The SDS Knowledge Portal consists of the following main components: 1) The SDS ontologies, stored in an Allegrograph RDF Store; 2) The SDS Ontology Server; and 3) The Web portal front end. At the start-up of the SDS Knowledge Portal, the Web application queries the SDS Ontology Server to retrieve a minimal set of information (labels and subsumption relations) about all the concepts, and populates the portal with this information. When the user accesses the portal and clicks on any concept of interest, the application sends a request to the Ontology Server to retrieve, parse, and display all information for this concept. Every time the portal submits a request to the SDS ontologies, the Ontology Server translates the request into a SPARQL query, and sends the query to the Allegrograph RDF Store to retrieve the relevant triples. Next, the Ontology Server parses the triples into a JSON serialization, and returns them to the front end. The Web portal then interprets the JSON string, renders the content, dynamically creates a page for the concept content, and displays the page in the user's browser. Figure 2 presents the system architecture and workflow of the SDS Knowledge Portal.

(Figure 2 about here)

\subsection{Browsing the SDS Knowledge Portal}

The SDS Knowledge Portal provides the user with several ways to browse the SDS ontologies. First, users can navigate the ontological hierarchy. Hierarchies are among the most common paradigms for Web navigation and form an intuitive starting point, especially 
for inexperienced users. The major components that were presented in Section 2.1 are reflected in the top categories of the hierarchy. Figure 3 shows these components, with the "problem type" taxonomy expanded.

(Figure 3 about here)

The ontology content can also be browsed by following non-taxonomic relations among concepts. As mentioned before, the concepts in the SDS ontologies are heavily interlinked via OWL object properties, facilitating the user's exploration of the portal. Browsing through such relations can be done through hyperlinks on the Portal (automatically generated base on the property defined in the ontology), highlighted in blue in Figure 3 above.

Browsing can also be done graphically. Each concept page on the portal contains a subgraph of the entire ontology graph showing the current concept and its relations. For reasons of performance and readability, the portal only displays nodes and links that constitute the definition of the concept. Figure 4 shows the graph for EMDS, a spatial decision support system. It defines EMDS by a software type, its relations to decision problem types, decision process steps, methods and case studies, and various system capability related specifications. The user can click on any node to navigate to that related concept.

(Figure 4 about here)

The user can also browse the ontology content alphabetically via a glossary that is automatically generated and populated from the ontologies; see Figure 5.

(Figure 5 about here)

As mentioned above, accessing information about SDS resources is one of the major services provided by the portal. Various categories of resources can be accessed via a 
Resources menu, and the instances of a resource category can be browsed alphabetically (Figure 6; they can also be searched, as explained below in Section 3.3).

(Figure 6 about here)

\subsection{Searching the SDS Knowledge Portal}

Besides browsing, the SDS resources can be searched by specifying a set of constraining criteria. The search criteria leverage the attributes and relations that are defined for a particular resource type in the SDS ontologies. For example, tools and models can be searched based on decision problem types targeted, relevant domain knowledge modeling areas, methods and techniques implemented, as well as system-related criteria such as platform or functional component. As the user selects values for the criteria, the search is performed by dynamically filtering ontology instances. Figure 7 shows the criteria against which tools and models can be searched, with the "required functional components" criterion value list expanded. It also shows the criteria that the user has specified so far, and the tools remaining on the list that satisfy the specified search criteria.

(Figure 7 about here)

The SDS resources can also be queried via a main search field on the portal via a semantic-enabled search, instead of regular keyword search, by performing query expansion (Bhogal et al. 2007). Query expansion is partly achieved by leveraging the synonym, abbreviation, and acronym information encoded for the concepts in the ontology. The search navigates the ontology graph by following the attributes and relations formally defined for particular resource types. A search for "AHP" (Figure 8) returns: the SDS method AHP (Saaty 1988), tools that implement AHP, case studies in which AHP was adopted, publications that include AHP-related discussions. The search involves the following steps: 1) Find the concept for the specified keyword ("AHP") in the ontology. In this case we find the concept Analytical Hierarchy Process (with "AHP" as the additional label); 2) Determine the type of AHP (it is a SDS method); 3) Invoke a search route predefined specifically for the concepts of the SDS methods type, which includes a limited set of predicates which the ontology server checks, such as the implements predicate for tools or the methodUsed 
predicate for case studies. 4) Query the RDF graph for related classes and instances based on this set of predicates. 5) Cluster the search results list based on SDS resource types.

(Figure 8 about here)

The semantic search leverages the subclass and sibling-class relations, as well as other transitive relations defined in the ontology, which is a more common means of expanding a search query. For example, the user may search for a tool that implements sensitivity analysis. In the SDS ontologies, sensitivity analysis is a method class with subclasses aspatial sensitivity analysis, spatial sensitivity analysis, and global sensitivity analysis. The first two subclasses have additional subclasses. When the user searches for tools that implement sensitivity analysis, the portal returns all the submethods of sensitivity analysis and the tools that implement any of the methods.

\section{Usage Scenarios for the Portal and Ontologies}

Due to its flexible user interface and broad range of resources, there are many different ways and reasons to use the SDS portal and ontologies, and they were set up with documentation, improved retrieval, and learning in mind. To demonstrate how our system supports these tasks, this section briefly discusses a retrieval and a learning use case.

\subsection{Learning Use Case}

Domain experts, researchers interested in applying SDS methods, and students interested in learning about SDS are likely to use the browsing and navigation interfaces of the portal. To give a concrete example, a student may want to learn about planning and decision workflows as an intuitive starting point for applying SDS as part of her research. ${ }^{2}$ The Explore the Ontology frame contains a collapsible list of topics starting from introductory topics over descriptions of methods up to useful resources. The frame gets automatically enlarged onmouse-over and the student navigates to the Planning And Spatial Decision Process Workflows section via Planning And Spatial Decision Process. The resulting page describes major workflow models and, among others, lists Steinitz's Framework. By following this hyperlink, the student accesses a new page that lists each of the six process phases, contains a

2 Readers are invited to follow the directions starting at http://www.spatial.redlands.edu/sds/. 
detail illustration, a reference to the paper by Steinitz et al., and links to more detailed descriptions of each phase. To assist the student in understanding whether this specific workflow model is relevant for her work, the page also lists application domains and decision problem types. As the researcher is interested in land use planning, Steinitz's framework seems appropriate. By following the domain link she can immediately learn about relevant software, e.g., IDRISI Land Change Modeler, or read about related case studies, such as Summit County Lower Blue Subbasin Master Plan. The description of this study provides detailed descriptions and links to relevant literature and lists lessons learned.

\subsection{Search Use Case}

In addition to browsing and navigating, some users may prefer a direct search. This is especially the case for those experts and researchers who are familiar with SDS and want to review appropriate methods and tools for their work. To give a concrete example, a researcher may be interested in those tools that were created with wildlife management and land use planning in mind and support suitability assessments. First, the researcher types in "wildlife" in the topmost right search box. This results in a categorized overview of all tools, data sources, case studies, and literature related to this query. As the researcher does not want to check all provided tools by hand, she selects the Tools and Models view from the Resources menu. The resulting page can be used to get an overview of all tools within a feature matrix or to filter them by specific properties defined in the SDS ontologies. For instance, by selecting Land Use Planning and Suitability Assessment as domain and problem type, respectively, the researcher receives a filtered list of tools. To further restrict the search, she filters tools by their costs and selects free tools to finally review the Refuge GAP software in more detail.

\section{Lessons Learned}

While developing the SDS ontologies we faced an interesting trade-off between representing knowledge and facilitating browsing and searching (see Section 2.3). We had to keep the information retrieval purpose in mind when designing the ontologies, while staying true to the underlying knowledge structure as much as possible. The main lessons we have learned from engineering these ontologies and the Knowledge Portal are as follows. First, the scope of coverage should be restricted to the immediate areas of expertise provided by the 
consortium, while links to external ontologies enrich the local specifications and reuse previous work on the Semantic Web. Second, when designing ontologies for a dynamic and growing research field by a heterogeneous consortium, following the rule of minimizing ontological commitments is crucial to ensure enough flexibility for future extensions. Third, labels and comments are not only key for documentation but are important to ease search and browsing through the knowledge portal. Fourth, and as suggested in the literature, the untangling, i.e., the introduction of multiple-taxonomic relations, should be done bottom-up by Semantic Web reasoners and not manually during ontology engineering. Fifth, inverse and transitive relations are key to improving navigation and inference, and keeping the size of the ontologies manageable. These relations should be inferred instead of defined. Finally, our work supports the claim (Baader et al. 2010) that when balancing knowledge representation and semantic search, higher expressivity is not necessarily the major concern.

\section{Conclusions and Future Work}

Spatial decision support is a dynamic and heterogeneous domain that benefits from a detailed description of its existing process workflows, methods and tools. Instead of simply choosing a set of parameters to classify SDS resources, we developed ontologies to capture the various aspects of spatial decision support ranging from decision problems, processes, methods and technology, over tools, models and data sources, to relevant case studies and literature. We collected a representative set of SDS resources and registered them against the parameters defined in SDS ontologies.

SDS knowledge is represented by a set of ontologies that were developed in common agreement among a large-scale consortium of researchers and practitioners from various subdomains of the field. Driven by the needs to document the body of knowledge in SDS, ease learning, and navigate through the portal, these ontologies provide a flexible conceptual framework for classification and characterization and are interlinked with other ontologies on the Semantic Web. By providing formal definitions, the ontologies become common vocabularies for the broader SDS community to facilitate re-usability and interoperability of SDS resources by reducing the risk of semantic mismatches. The ontologies may also serve as the basis for intelligent SDS applications that provide guidance for tasks such as configuring a SDS system by suggesting appropriate methods, tools, and models based on the parameters of a specific decision problem. Our framework also contributes to the establishment of a standard for describing SDS services that can be used in Web registries to 
facilitate service interoperability and chaining as proposed by the Open Geospatial Consortium.

The SDS ontology development work will continue for the foreseeable future. Currently there is interest within the user community to deepen the knowledge in the ontology for targeted knowledge domains, and we have started to do this for the domain of urban planning. Given our purpose of serving SDS, we are only including concepts that are pertinent to planning and decision making in this domain. Besides deeper development of a particular ontology branch, many existing concepts have very skeletal definitions that can benefit from further development. Another important area of future work is related to handling dialect differences across user communities, such as scientists vs. land managers vs. designers. Although we will have a standard label for a concept, we can accommodate alternate word usage by presenting the preferred term for this concept in a view tailored for a particular user community, analogous to using different language labels in the ontology. An important future direction is to leverage more of the logical structure in the ontology for reasoning (e.g., the relations between decision problem types and decision process workflows, methods, and various resources), and to develop more intelligent applications that provide automatic guidance to the user in selecting suitable SDS resources depending on the characteristics of their actual decision problems. As proposed by the SEAL approach (Maedche et al. 2003), integrating semantic similarity to improved navigation and retrieval (Janowicz et al. 2011) is a next step for the portal. With the Allegrograph triple store and SPARQL support in place, we are also considering serving the instance data as Linked Data for science and education. Finally, a systematic user review on both the SDS ontologies and the Knowledge Portal would be useful to obtain more insight for improving the content as well as usability of the Portal.

\section{Acknowledgements}

Naicong Li's work was supported in part by funds provided by the U.S. Army Research Laboratory and the U.S. Army Research Office under grant number W911NF-07-1-0392. 


\section{References}

Armstrong M P 1993 Perspectives on the development of group decision support systems for locational problem solving, Geographical Systems 1: 69-81

Baader F, Lutz C, and Turhan A Y 2010 Small is again Beautiful in Description Logics. KI Künstliche Intelligenz, 24(1): 25-33

Batty M 2008 Planning Support Systems: progress, predictions, and speculations on the shape of things to come. In Brail R. (ed) Planning Support Systems for Cities and Regions. Lincoln Institute of Land Policy, Cambridge, Massachusetts, 3-30

Bhogal J, Macfarlane A and Smith P A 2007 Review of ontology-based query expansion. Information Processing and Management 43: 866-886

Densham P J 1991 Spatial decision support systems. In Maguire D J, Goodchild M F and Rhind D W (eds) Geographical Information Systems: Principles and Applications, New York: John Wiley and Sons, 403-412

De Roure D and Hendler J A 2004 E-Science: the grid and the Semantic Web. Intelligent Systems, IEEE. 19(1): 65-71

Fernandez M, Gomez-Perez A and Juristo N 1997 METHONTOLOGY: from Ontological Art towards Ontological Engineering. AAAI Symposium 1997: 33-40

Goodchild M F and Glennon J A 2008 Representation and computation of geographic dynamics. In Hornsby K S and Yuan M (eds) Understanding Dynamics of Geographic Domains, Boca Raton: CRC Press: 13-30

Harvey F, Kuhn W, Pundt H, Bishr Y and Riedemann C 1999 Semantic interoperability: A central issue for sharing geographic information. The Annals of Regional Science, 33: 213-232

Jankowski P and Nyerges T 2001 Geographic Information Systems for Group Decision Making. New York: Taylor and Francis

Jankowski P, Nyerges T, Robischon S, Ransey K and Tuthill D 2006 Design considerations and evaluation of a collaborative, spatio-temporal decision support system. Transactions in GIS 10(3): 335-354

Janowicz K, Raubal, M, and Kuhn, W 2011 The semantics of similarity in geographic information retrieval. Journal of Spatial Information Science 2: 29-57

Leung Y 1997 Intelligent Spatial Decision Support Systems. Berlin, Springer-Verlag

Ligmann-Zielinska A and Jankowski P A 2008 Framework for Sensitivity Analysis in Spatial Multiple Criteria Evaluation. In GIScience 2008, LNCS 5266, Cova T J et al. (Eds) Springer-Verlag Berlin: 217-233

Maedche A, Staab S, Stojanovic N, Studer R and Sure Y 2003 SEmantic portAL: The SEAL Approach. In Fensel D, Hendler J A, Lieberman H, and Wahlster W (eds) Spinning the Semantic Web, MIT Press: 317-359

Malczewski J 1999 GIS and Multicriteria Decision Analysis. New York: John Wiley and Sons

Malczewski J 2006 GIS-based multicriteria decision analysis: A review of the literature. International Journal of Geographical Information Science 20(7): 703-726

Raskin R G and Pan M J 2005 Knowledge representation in the Semantic Web for Earth and Environmental Terminology (SWEET). Computers and Geosciences 31: 1119-1125 
SDS Knowledge Portal, http://www.spatial.redlands.edu/sds/ontology/?n=SDSSAbout:SDS

Saaty T L 1988 The Analytical Hierarchy Process: Decision Making for Leaders in a Complex World. Pittsburgh: University of Pittsburgh Press

Shvaiko P and Euzenat J 2008 Ten challenges for ontology matching on the move to meaningful Internet systems: OTM 2008. Lecture Notes in Computer Science Volume 5332/2008, Berlin: Springer: 1164-1182

Sieber R 2006 Public participation geographic information systems: A literature review and framework. Annals of the Association of American Geographers 96(3): 491-507

Simon H A 1960 The New Science of Management Decision. New York: Harper \& Row

Steinitz C A 1990 framework for theory applicable to the education of landscape architects (and other environmental design professionals). Landscape Journal 9: 136-143

Spatial Decision Support Consortium, http://www.spatial.redlands.edu/sds/consortium/

Suominen O, Viljanen K, and Hyvönen E. 2007 User-centric faceted search for semantic portals. In Franconi E, Kifer M and May W (eds) ESWC '07 Proceedings of the 4th European conference on The Semantic Web: Research and Applications. Springer LNCS 4519: $356-370$

Zhu X and Healey R 1992 Towards intelligent spatial decision support: integrating geographical information systems and expert systems. In Proceedings of the GIS/LIS'92: $877-886$ 
List of figures

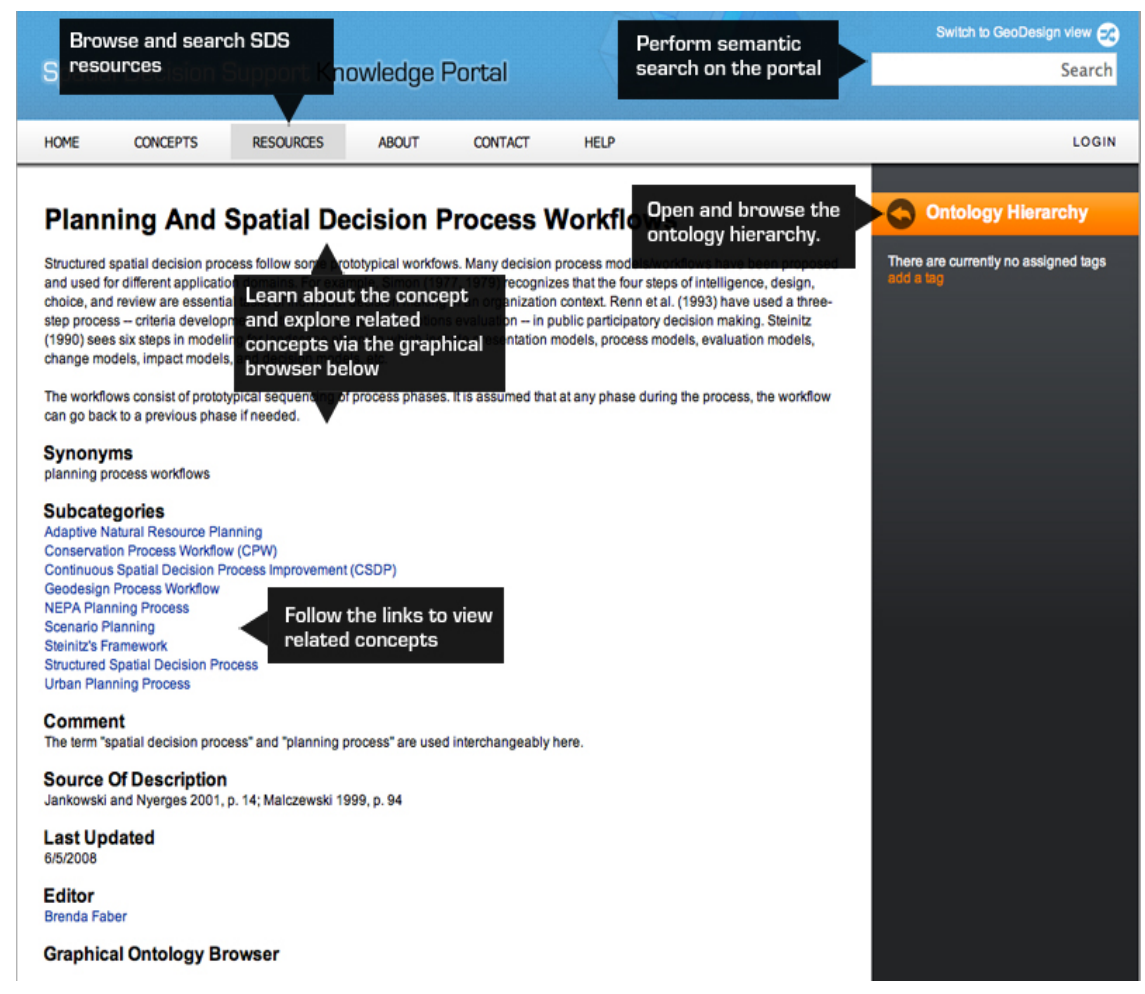

Figure 1. The main functional components of the SDS Knowledge Portal. 


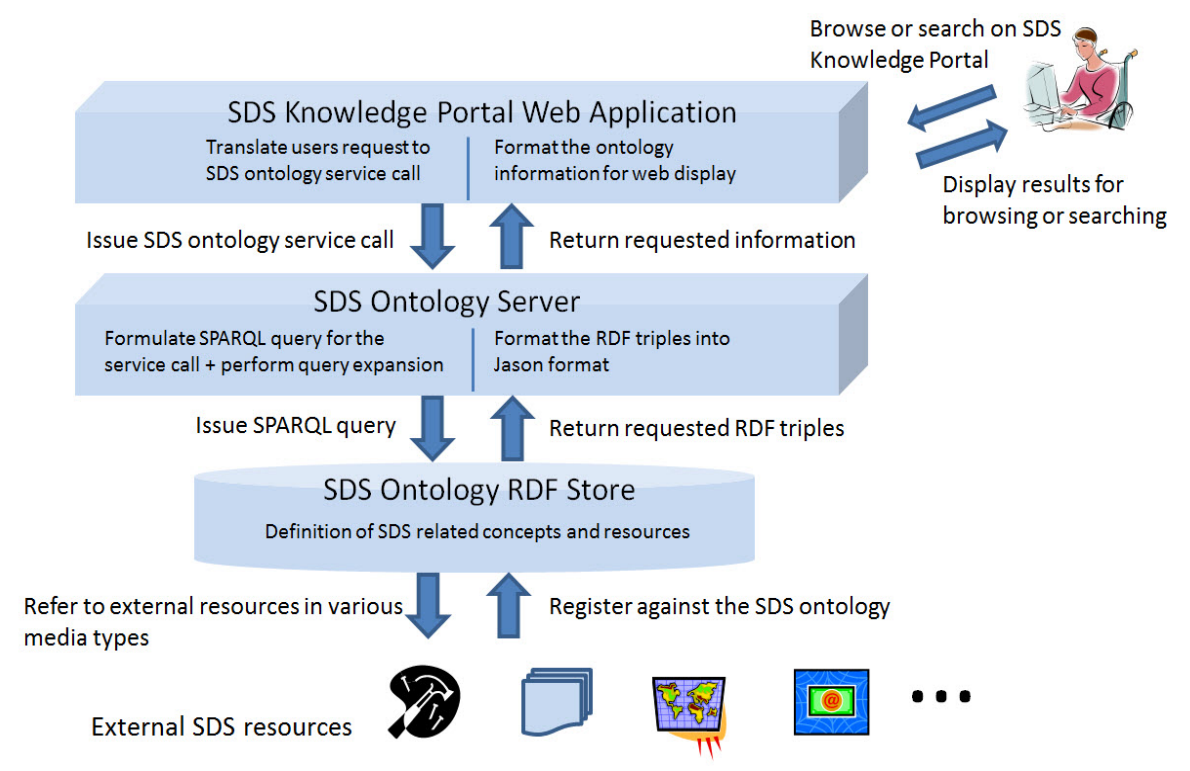

Figure 2. System architecture and workflow of the SDS Knowledge Portal 


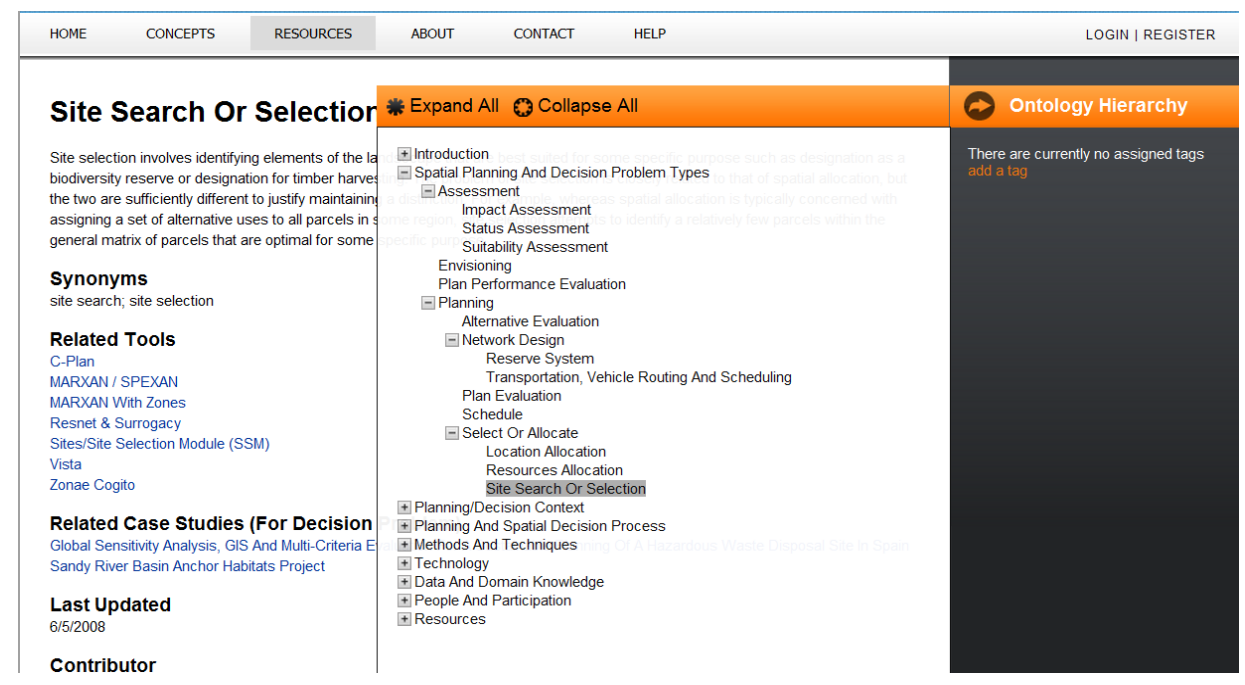

Figure 3. Browsing the ontology through the ontology hierarchy and relations among the concepts and instances 


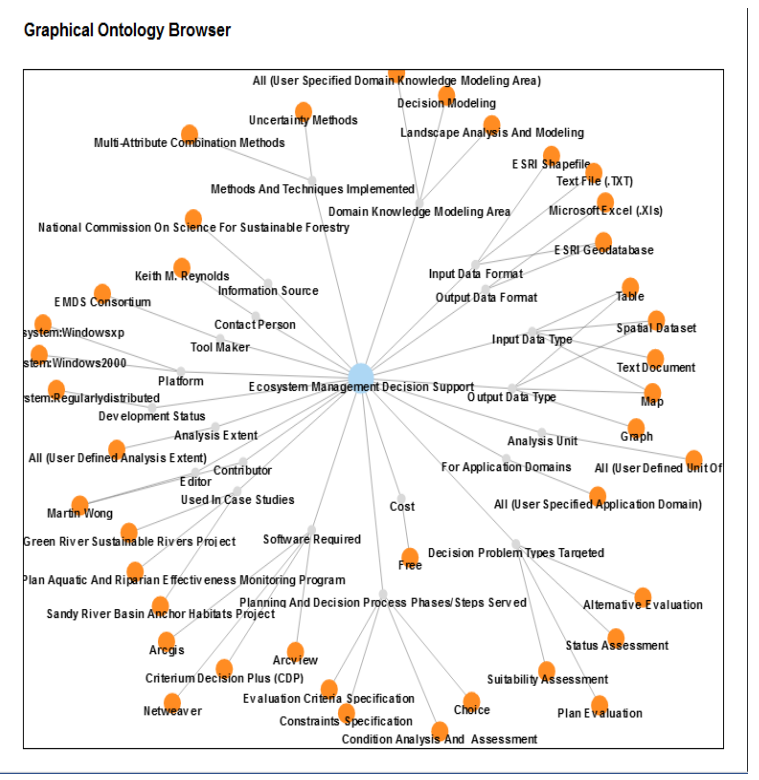

Figure 4. Graphical ontology browsing via relationships 


\begin{tabular}{l} 
Spatial Decision Support Knowledge Portal \\
\hline HOME CONCEPTS RESOURCES ABOUT CONTACT HELP \\
\hline Concepts Glossary
\end{tabular}

Figure 5. Browsing the Glossary 


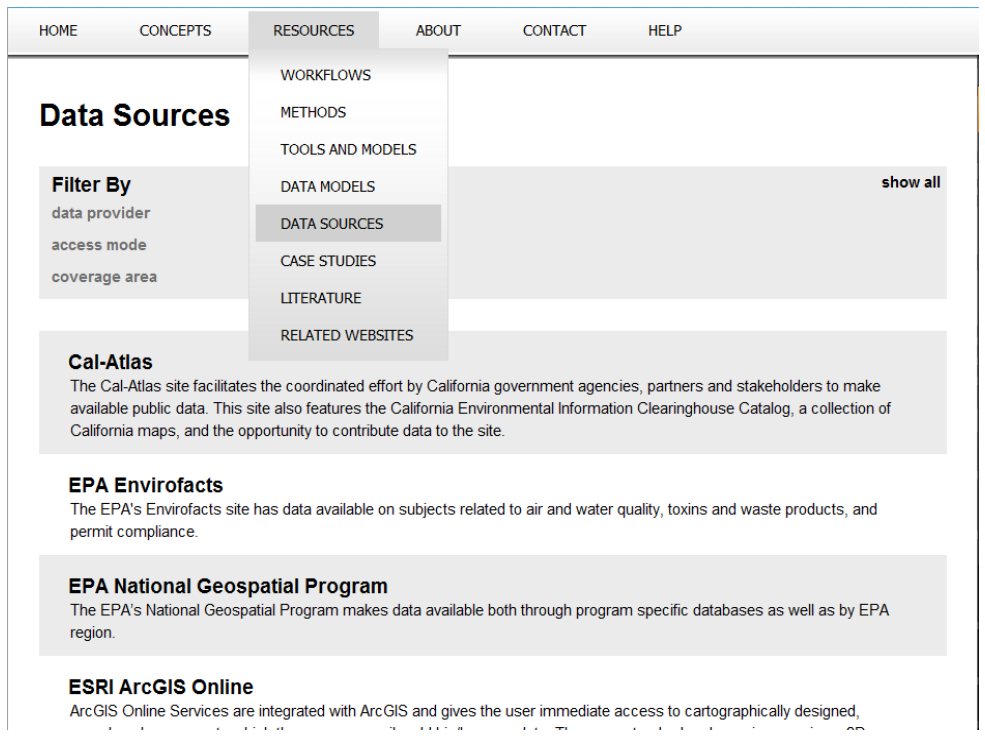

Figure 6. Browsing the instances of data sources 


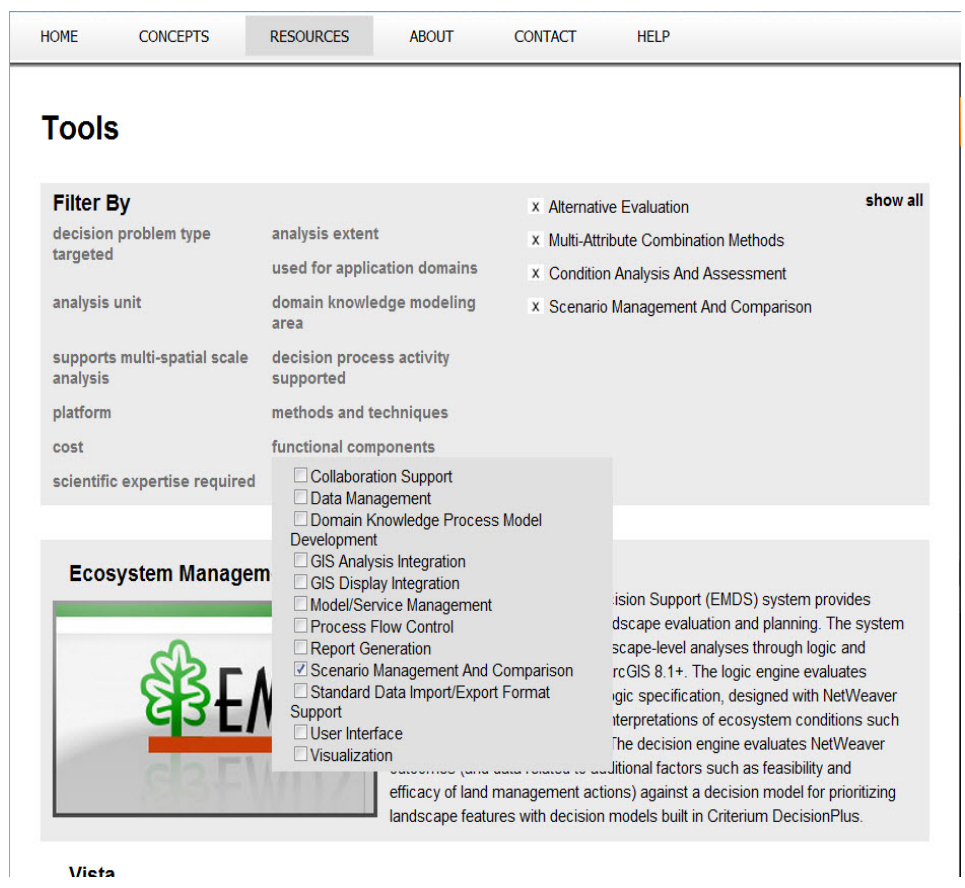

Figure 7. The multi-criteria tools and models search 


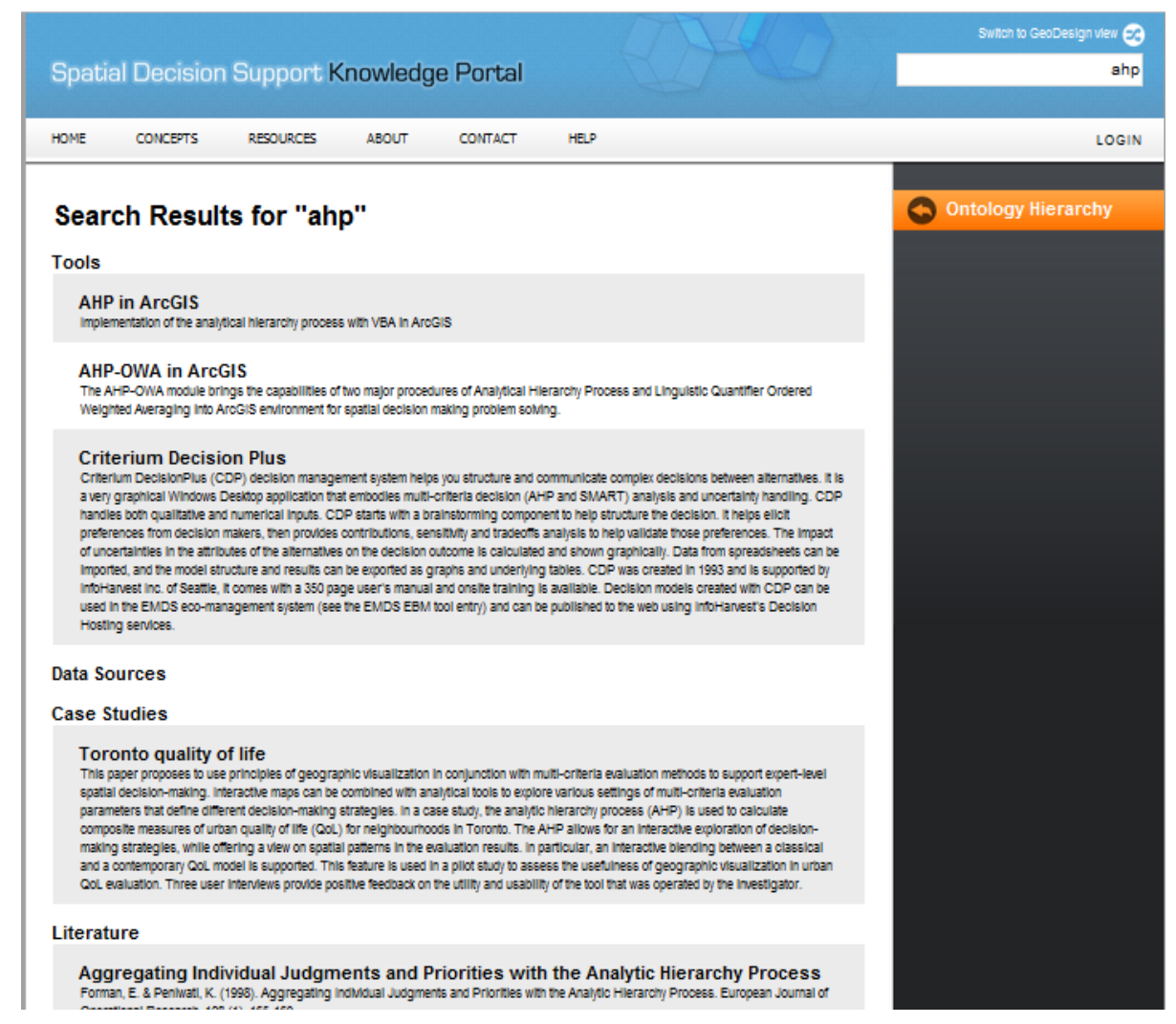

Figure 8: Semantic search 